\begin{tabular}{|c|c|}
\hline Citation & $\begin{array}{l}\text { Kang Cui, Kunal S. Mali, Oleksandr Ivasenko, Dongqing Wu, Xinliang Feng, } \\
\text { Michael Walter, Klaus Müllen, Steven De Feyter, Stijn F. L. Mertens } \\
\text { Squeezing, Then Stacking: From Breathing Pores to Three- } \\
\text { Dimensional lonic Self-Assembly under Electrochemical Control } \\
\text { Angewandte Chemie International Edition, 2014, 53, } 12951-12954\end{array}$ \\
\hline Archived version & $\begin{array}{l}\text { Author manuscript: the content is identical to the content of the published } \\
\text { paper, but without the final typesetting by the publisher }\end{array}$ \\
\hline Published version & $\begin{array}{l}\text { insert link to the published version of your paper } \\
\text { http://dx.doi.org/10.1002/anie.201406246 }\end{array}$ \\
\hline Journal homepage & $\begin{array}{l}\text { insert link to the journal homepage of your paper } \\
\text { http://onlinelibrary.wiley.com/journal/10.1002/(ISSN) } 1521-3773 \text {. }\end{array}$ \\
\hline Author contact & $\begin{array}{l}\text { your email steven.defeyter@kuleuven.be } \\
\text { your phone number }+32(0) 16327921\end{array}$ \\
\hline IR & url in Lirias https://lirias.kuleuven.be/handle/123456789/477342 \\
\hline
\end{tabular}




\title{
Squeezing, then Stacking: from Breathing Pores to 3-Dimensional Ionic Self-Assembly under Electrochemical Control***
}

\author{
Kang Cui, Kunal S. Mali, Oleksandr Ivasenko, Dongqing Wu, Xinliang Feng, Michael Walter, \\ Klaus Müllen, Steven De Feyter, * and Stijn F. L. Mertens*
}

\begin{abstract}
We demonstrate the spontaneous and reversible transition between two- and three-dimensional self-assembly of a supramolecular system at the solid-liquid interface under electrochemical conditions, using in situ scanning tunneling microscopy. By tuning the interfacial potential, we can selectively organize our target molecules in an open porous pattern, fill these pores to form an auto-host-guest structure, or stack the building blocks in a stratified bilayer. Using a simple electrostatic model, we rationalize which charge density is required to enable bilayer formation, and conversely, which molecular size/charge ratio is necessary in the design of new building blocks. Our results may lead to a new class of electrochemically controlled dynamic host-guest systems, artificial receptors and smart materials.
\end{abstract}

$\boldsymbol{A}_{\mathrm{lt}}$ hough generally highly developed, ${ }^{[1]}$ molecular self-assembly at the solid-liquid interface still faces several challenges, in particular related to reaching into the third dimension. To the best of our knowledge, adaptable surface-based supramolecular architectures perpendicular to the surface have been only achieved thus far by the

[*] K. Cui, Dr. K. Mali, Dr. O. Ivasenko, Prof. S. De Feyter, Dr. S. F. L. Mertens

Department of Chemistry, KU Leuven

Celestijnenlaan 200F, 3001 Leuven (Belgium)

E-mail: steven.defeyter@chem.kuleuven.be (S. De Feyter); stmerten@gmail.com (S. F. L. Mertens)

\section{Dr. S. F. L. Mertens}

Institut für Angewandte Physik, TU Wien

Wiedner Hauptstraße 8-10/E134, 1040 Wien (Austria)

Dr. M. Walter

Freiburger Materialforschungszentrum, Universität Freiburg

79104 Freiburg i. Br. (Germany)

Fraunhofer IWM, 79108 Freiburg i. Br. (Germany)

Dongqing Wu, Dr. X. Feng, Prof. K. Müllen

Max Planck Institute for Polymer Research 55128 Mainz (Germany)

[**] K.C. acknowledges the receipt of a PhD scholarship of the China Scholarship Council, and S.F.L.M. the receipt of a Marie Curie European reintegration grant. This work was further supported by the Fund for Scientific ResearchFlanders (FWO-Vlaanderen) and by the European Research Council under the European Union's Seventh Framework Programme (FP7/2007-2013)/ERC Grant Agreement No. 340324, Nanograph@LSI, and advanced ERC grants Nanograph and OxideSurfaces. We thank FZJülich for computational resources.

Supporting information for this article is available on the WWW under http://dx.doi.org/10.1002/anie.201xxxxxx. use of multiple building blocks or tectons, ${ }^{[2 a-f]}$ or by charge reversal of the substrate through anion adsorption. ${ }^{[2 \mathrm{~g}]}$ In this paper, we present a system based on an organic salt (polyaromatic cation + perchlorate anion) whose rich supramolecular features allow us to do exactly that with a single compound and an unmodified substrate: by tuning the electrochemical potential, we can assemble the tectons into a porous structure, stretch these pores to accommodate another molecule as a guest, or stack them into an ordered bilayer in a controlled manner. The changes are fully reversible and could form the basis of electrochemically controlled dynamic host-guest systems and 3D structures such as artificial receptors. ${ }^{[3]}$

Our target molecule, 9-phenylbenzo[1,2]quinolizino[3,4,5,6-fed] phenanthridinylium perchlorate (Figure 1), and further denoted as $\mathrm{PQPClO}_{4}$, is a charged discoid polycyclic aromatic compound which is known to stack efficiently into columnar structures in the solid state. ${ }^{[4]}$ The compound occurs as a salt and its composing ions are therefore expected to respond strongly to the effects of interfacial potential $^{[5]}$ and the electrostatic forces that govern ionic selfassembly. ${ }^{[6]}$ The extended aromatic system, on the other hand, is flat and therefore prone to strong interaction with a gold substrate by virtue of its $\pi$-electrons. ${ }^{[7]}$ As we were not interested at this stage in competitive adsorption between the target molecule and the supporting electrolyte, all experiments were performed in perchloric acid solution, whose anion does not specifically adsorb on gold.

The cyclic voltammogram (see Supporting Information) of the modified $\mathrm{Au}(111)$ electrode in $0.1 \mathrm{HClO}_{4}$ was featureless, indicating the absence of redox processes and the slow timescale of phase transitions, which are known to cause sizeable capacitive peaks when reorientation is fast compared to scan rate. ${ }^{[8]}$<smiles></smiles>

Figure 1. Molecular structure of $\mathrm{PQPCIO}_{4}$

Figure 2A shows a high-resolution STM image obtained at a substrate potential of $0.6 \mathrm{~V} v s$ RHE, where a highly ordered and visibly porous structure covers the surface. A careful analysis of the image and of the allowed intermolecular orientation (see Supporting Information) reveals that the bright spot corresponds to the fused aromatic system of $\mathrm{PQP}^{+}$, so that a molecular arrangement as shown in Fig. 2B emerges: six $\mathrm{PQP}^{+}$cations display a 6-fold rotational 
symmetry in which the lone phenyl ring is located at the bay position of its neighboring cation, around a $0.9-\mathrm{nm}$ pore. The structure is reminiscent of the hexagonal arrangement observed on HOPG, ${ }^{[9]}$ although in the latter case no electrochemical control was exerted. Also akin to this study, the perchlorate counter ions were never directly observed in the STM images. For reasons of electroneutrality, it is essential that they occupy positions close to the $\mathrm{PQP}^{+}$cation adlayer, although they may at the same time be highly mobile. Our DFT calculations predict effective screening of the electrostatic interactions by the aqueous environment, resulting in only $14 \mathrm{~kJ} \mathrm{~mol}^{-}$ 1 binding energy between the ions and endorsing their facile separation (see also Supporting Information).
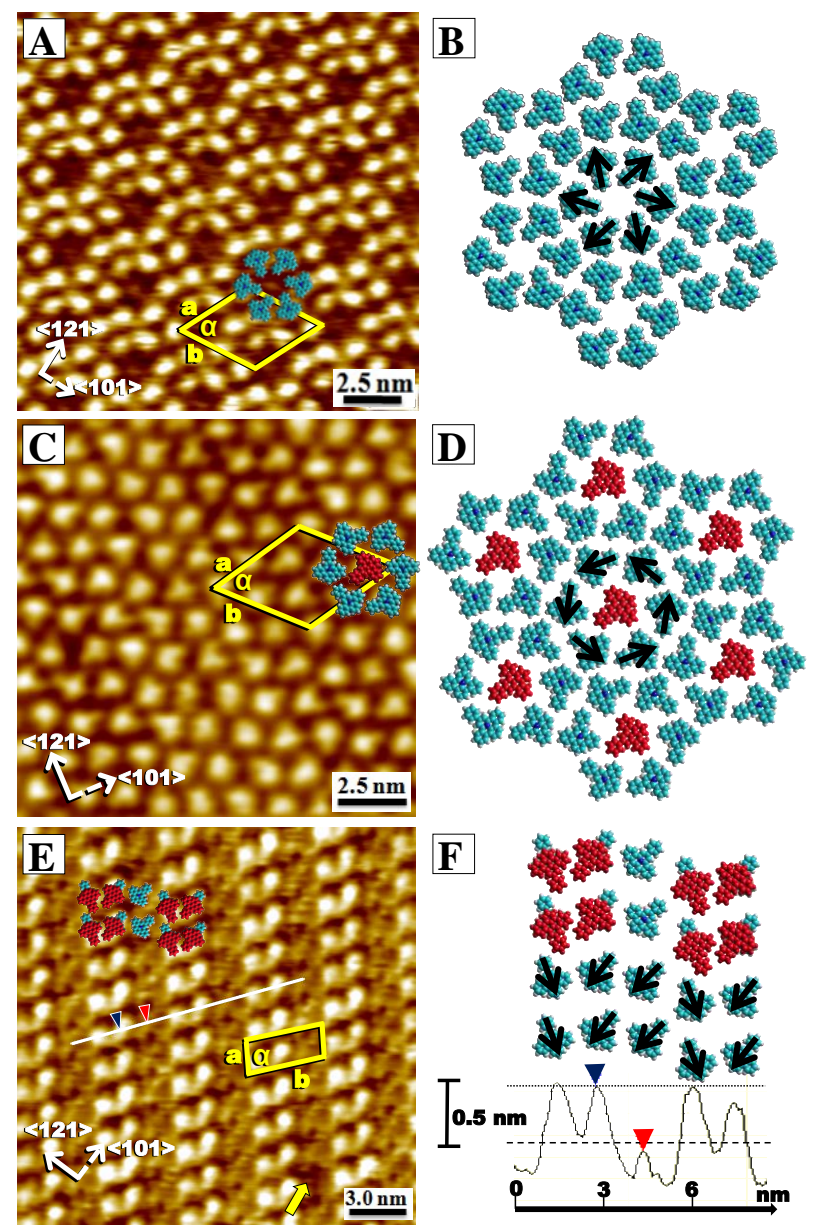

Figure 2. (A) High-resolution STM image of porous pattern I for $\mathrm{PQPClO}_{4}$-modified Au(111) in $0.1 \mathrm{M} \mathrm{HClO}_{4}$. Substrate potential $E_{\mathrm{s}}=$ $0.6 \mathrm{~V}$ vs RHE, tip bias voltage $U_{b}=-0.62 \mathrm{~V}$, tunneling current $l_{\mathrm{t}}=1.0$ $\mathrm{nA}$. Unit cell parameters $a=b=(3.7 \pm 0.2) \mathrm{nm}, \alpha=(59 \pm 2)^{\circ}$. Pore van der Waals area $A_{1}=2.5 \mathrm{~nm}^{2}$. (B) Tentative model for the assembly. (C) High-resolution STM image of filled porous auto-host-guest pattern II. $E_{\mathrm{s}}=0.4 \mathrm{~V}, U_{\mathrm{b}}=-0.61 \mathrm{~V}, I_{\mathrm{t}}=1.0 \mathrm{nA}$. Unit cell parameters $a=b=$ $(3.8 \pm 0.2) \mathrm{nm}, \alpha=(60 \pm 2)^{\circ}$. Pore van der Waals area $A_{\|}=3.9 \mathrm{~nm}^{2}$. (D) Tentative model for the molecular arrangement, showing the host lattice (blue) and guest molecules (red), and rotation compared to pattern I. (E) High-resolution STM image of bilayer structure IIIA. $E_{\mathrm{s}}=$ $0.15 \mathrm{~V}, U_{b}=-0.5 \mathrm{~V}, I_{t}=1.0 \mathrm{nA}$. Unit cell parameters $a=(1.9 \pm 0.1)$ $\mathrm{nm}, b=(4.3 \pm 0.2) \mathrm{nm}, \alpha=(78 \pm 2)^{\circ}$. The yellow arrow indicates a missing molecule in the 1st layer. $(F)$ Tentative model for structure IIIA, showing first layer (blue) and second layer (red) molecules, and crosssectional profile along the white line in Panel $\mathrm{E}$.
On making the substrate potential $200 \mathrm{mV}$ more negative, at first sight a major change in molecular packing is observed in which the initial porosity seems to have disappeared, Figure 2C. A closer inspection of the high-resolution image reveals that the tectons forming the original porous structure are still arranged in a hexagonal pattern, but are rotated to enlarge the pore and accommodate a further $\mathrm{PQP}^{+}$cation in the center in an auto-host-guest mechanism. ${ }^{[10]}$ The more than $50 \%$ increase in van der Waals pore area from structure I to II is almost entirely accomplished by a $90^{\circ}$ rotation of the $\mathrm{PQP}^{+}$ cation, as the center-to-center distance increases by only $12 \%$. Even though the new unit cell is slightly larger, the overall molecular density has increased by $10 \%$ in pattern II, at 0.55 molecules $\mathrm{nm}^{-2}$.

In Figure 2C, and especially in large-scale images (see Supporting Information), the herringbone reconstruction of the $\mathrm{Au}(111)$ substrate is visible through the adlayer, ${ }^{[11 \mathrm{a}]}$ indicating that the adsorption is not strong enough to lift the reconstruction. Further, it exemplifies that the supramolecular arrangement is robust and relatively insensitive to the exact adsorption sites available. We did not observe any variation in intermolecular distance depending on the position of the adsorbates on the reconstructed surface, although mild orientational preferences in the $\langle 121\rangle$ direction are expressed. ${ }^{[11 \mathrm{~b}]}$ This suggests that the adsorption is essentially incommensurate with the gold substrate, and that the layer morphology is primarily governed by intermolecular interactions.

A further negative shift of the substrate potential to $0.15 \mathrm{~V}$ leads to a fundamentally different packing (pattern IIIA, Figure 2E) in which a highly compact lamellar adlayer of $\mathrm{PQP}^{+}$cations in contact with the Au substrate is covered by double rows of additional $\mathrm{PQP}^{+}$ cations in a second layer. The second layer has the same registry as the first, and leaves troughs through which the first layer can be directly observed. A second polymorph of this pattern, IIIB, with slightly different lattice parameters, was also found (see Supporting Information). The double layer characteristic of pattern IIIA is confirmed by the cross-sectional profile (Figure $2 \mathrm{~F}$ ) which indicates a doubling of the apparent height at the location of the bright rows, while identification of the tectons in the first layer is facilitated by the occurrence of a missing $\mathrm{PQP}^{+}$cation indicated by a yellow arrow in the lower right of Figure 2E.

Importantly, $\mathrm{PQP}^{+}$bilayer structures were never observed in conventional solid/liquid STM studies on HOPG ${ }^{[9]}$ although similar bias values were used, which suggests that electrochemical control is essential to achieve 3D assembly. Based on high-resolution STM images and the elucidated crystal structure of PQP stacks in solution, ${ }^{[4 c]}$ we propose the model in Figure $2 \mathrm{~F}$, in which two $\mathrm{PQP}^{+}$ cations are packed in a cofacial and antiparallel arrangement. By stacking into two layers, the total packing density increases further to 0.62 molecules $\mathrm{nm}^{-2}$, although the first layer (with 0.37 molecules $\mathrm{nm}^{-2}$ ) has again relaxed compared to patterns I and II, which is sterically favorable.

Table 1. Substrate charge compensation by $\mathrm{PQP}^{+}$adlayers

\begin{tabular}{lllll}
\hline Structure & $\begin{array}{l}E_{\mathrm{s}} \\
{[\mathrm{V} \text { vs }} \\
\text { RHE }]\end{array}$ & $\begin{array}{l}|q(\mathrm{Au})|^{[\mathrm{a}]} \\
{\left[\mu \mathrm{C} \mathrm{cm}{ }^{-2}\right]}\end{array}$ & $\begin{array}{l}\square{ }^{[\square} \Gamma^{[\mathrm{b}]} \\
{\left[\mathrm{mol} \mathrm{cm}^{-2}\right]}\end{array}$ & $\begin{array}{l}q(\mathrm{PQP})^{[\mathrm{cc}]} \\
{\left[\mu \mathrm{C} \mathrm{cm}^{-2}\right]}\end{array}$ \\
\hline I & 0.6 & 0.5 & 8.5 & 7.4 \\
II & 0.4 & 5.0 & 9.1 & 7.9 \\
bilayer IIIA & 0.15 & $\mathbf{7 . 2}$ & $6.2^{[\mathrm{d}]}$ & $\mathbf{5 . 4}$ \\
bilayer IIIB & 0.15 & $\mathbf{7 . 2}$ & $7.5^{[\mathrm{d}]}$ & $\mathbf{6 . 5 ^ { [ \mathrm { d } | }}$ \\
\hline
\end{tabular}


[a] Formal substrate charge density. [b] Surface coverage. [c] Cation adlayer charge density. [d] First layer only.

In order to rationalize the bilayer formation, we consider the extent of surface charge compensation by the cation adlayer. From the potential of zero charge $(\mathrm{pzc}=0.62 \mathrm{~V})^{[11 \mathrm{c}, \mathrm{d}]}$ of the reconstructed $\mathrm{Au}(111)-(22 \times \sqrt{3})$ surface in dilute perchloric acid solutions and the capacitive current in cyclic voltammetry (Fig. S1), we can formally estimate the charge density on the gold surface for the potentials where the different adlayers are observed (for full details, see Supporting Information).

From the data in Table 1, the two single-layer structures I and II overcompensate the substrate charge to varying degrees, as in both cases $q\left(\mathrm{PQP}^{+}\right)>|q(\mathrm{Au})|$, which indicates that the adsorption is $\pi$ electron driven (the adsorption energy is on the order of $200 \mathrm{~kJ} \mathrm{~mol}^{-}$ $\left.{ }^{1}{ }^{[7]}\right)$. This also explains why for positive surface charges (i.e., when $E_{\mathrm{s}} \geq 0.8 \mathrm{~V}$ ), the adlayer amorphizes (see Supporting Information). For sufficiently negative potentials, the negative charge density of the substrate is no longer fully compensated by a single-layer cationic adsorbate (bold entries in Table 1), in agreement with the emergence of bilayer structures.

Conversely, we can estimate which footprint/charge ratio is required in the design of new charged organic molecules to enable the possibility of electrochemical switching between single and bilayer self-assembly. Assuming the same potential window as observed here, and a similar interfacial capacitance, we arrive at a threshold footprint of $1.6 \mathrm{~nm}^{2}$ for monocationic species, below which no bilayer formation is expected. Crucial is that the monolayer charge must not fully compensate the substrate surface charge, which may explain why spontaneous bilayer formation has not been observed previously: charged porphyrins, although studied extensively, often carry up to 4 charges per molecule ${ }^{[5 b-d]}$ and will achieve full charge compensation with (sub)monolayer coverage up to the limits of the available potential window.

A further requirement for bilayer formation is that the repulsive energy between the cations is not too high. The formation of a stacking motif in the crystal structure ${ }^{[4]}$ indicates that such a configuration is energetically favored. Our DFT calculations reveal a binding energy of $151 \mathrm{~kJ} \mathrm{~mol}^{-1}$ between two $\mathrm{PQPClO}_{4}$ units in aqueous environment in the configuration depicted in Figure 3B. A Bader charge analysis ${ }^{[12]}$ shows that the positive charge is distributed over the entire molecule (see Figure 3A)) enabling this strong intermolecular interaction, which is confirmed by crystallographic and NMR data. ${ }^{[4]}$
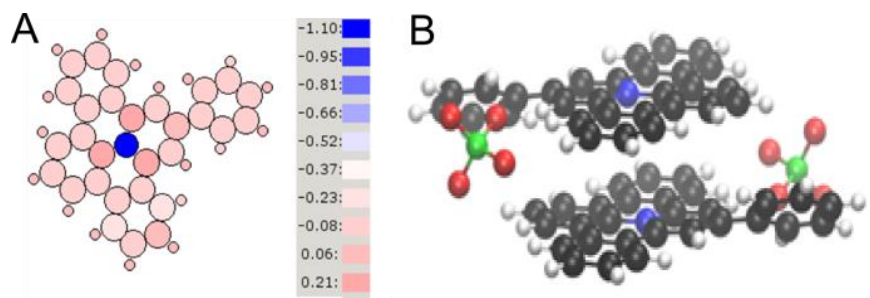

Figure 3. (A) Charge analysis of $\mathrm{PQP}^{+}$and $(\mathrm{B})$ relaxed structure of the $\mathrm{PQPClO}_{4}$ dimer (C: black, $\mathrm{N}$ : blue, $\mathrm{H}$ : white, O: red, Cl: green).
The formation of the bilayer phase of $\mathrm{PQPClO}_{4}$ is a relatively slow process, much more so than the transition between porous structures I and II on changing the substrate potential (see Supporting Information). As always, the thermodynamically most stable structure is dictated by a balance of all forces involved (electrostatic, steric, van der Waals), and can be hindered by kinetic trapping. Only after approximately $2 \mathrm{~h}$, the formation of the bilayer phase was completed. One possible reason, apart from the major redistribution of supramolecular building blocks when going from adlayer II to bilayer IIIA, is that the electrostatic force at the location of the second layer is considerably smaller than in the first layer, as shielding by the first layer cations occurs.

It is conceivable that a further increase of negative charge density on the Au substrate, brought about by a more negative substrate potential, leads to a full second layer or, alternatively, to molecular stacking in a 3rd layer. The potential window of the substrateelectrolyte combination used here, however, did not allow us to investigate this possibility.

In summary, we have demonstrated for the first time reversible switching between 2D and 3D self-assembly of charged organic molecules at an unmodified electrochemical solid-liquid interface. We anticipate that the rational design and synthesis of organic molecules along the principles outlined here will provide substantial advances in fields ranging from artificial receptors for highly specific sensing to smart materials.

\section{Experimental Section}

$\mathrm{PQPClO}_{4}$ was synthesized according to published procedures. ${ }^{[4 \mathrm{c}]}$ Electrolyte solutions were prepared from ultrapure $70 \% \mathrm{HClO}_{4}$ (Merck Suprapur) and high purity water (Milli-Q, Millipore, $18.2 \mathrm{M} \Omega \mathrm{cm}$, TOC < $5 \mathrm{ppb}$ ). The experiments were carried out in an inert atmosphere (Argon 5.0, Air Liquide). The substrate was a Au(111) single crystal (miscut $<0.1^{\circ}$, Mateck GmbH, Germany). ECSTM experiments were performed with an instrument designed and constructed in the group of Prof. Klaus Wandelt (University of Bonn). ${ }^{[13]}$ A platinum wire was used as a quasi-reference electrode and all potentials given in the text are rescaled to a reversible hydrogen electrode (RHE). To deposit $\mathrm{PQPCIO}_{4}$ on the gold substrate, a drop of saturated ethanol solution was allowed to evaporate on the previously flame-annealed $\mathrm{Au}(111)$ surface, followed by copious rinsing with ethanol and drying under Ar. The STM tips were electrochemically etched tungsten wires and were coated with molten polymer to minimize Faradaic currents. STM images were analyzed using SPIP 5.1 (Image Metrology) software. Density functional theory calculations were performed within the projector augmented wave method as implemented in the GPAW package ${ }^{[14,15]}$ van der Waals interactions were modeled as proposed by Tkatchenko and Scheffler. ${ }^{[16]}$ The aqueous environment was considered within a polarizable continuum model recently implemented by us. ${ }^{[17]}$

Received: ((will be filled in by the editorial staff)) Published online on ((will be filled in by the editorial staff))

Keywords: 3D self-assembly · porous networks · auto-host-guest system · organic salt · electrochemical scanning tunneling microscopy

[1] a) J. V. Barth, G. Costantini, K. Kern, Nature 2005, 437, 671-679; b) S. De Feyter, F. C. De Schryver, Chem. Soc. Rev. 2003, 32, 139-150; c) L. 


\section{Angewandte}

J. Wan, Acc. Chem. Res. 2006, 39, 334-342; d) J. A. Theobald, N. S Oxtoby, M. A. Phillips, N. R. Champness, P. H. Beton, Nature 2003, 424, 1029-1031.

[2] a) M. O. Blunt, J. C. Russell, M. del Carmen Giménez-López, N. Taleb, X. Lin, M. Schröder, N. R. Champness, P. H. Beton, Nature Chem. 2011, 3, 74-78; b) R. Madueno, M. T. Räisänen, C. Silien, M. Buck, Nature 2008, 454, 618-621; c) S. Yoshimoto, E. Tsutsumi, Y. Honda Y. Murata, M. Murata, K. Komatsu, O. Ito, K. Itaya, Angew. Chem. Int Ed. 2004, 43, 3044-3047; d) D. Bonifazi, A. Kiebele, M. Stohr, F. Y. Cheng, T. Jung, F. Diederich, H. Spillmann, Adv. Funct. Mater. 2007, 17, 1051-1062; e) R. Wen, C. J. Yan, H. J. Yan, G. B. Pan, L. J. Wan, Chem. Commun. 2011, 47, 6915-6917; f) J. Y. Gu, T. Chen, L. Wang, W. L. Dong, H. J. Yan, D. Wang, L. J. Wan, Langmuir 2014, 30, 35023506; g) M. Röefzaad, M. Jiang, V. Zamlynny, K. Wandelt, J. Electroanal. Chem. 2011, 662, 219-228.

[3] Artificial Receptors for Chemical Sensors (Eds: V. M. Mirsky, A. K. Yatsimirsky), Wiley-VCH, Weinheim, 2011.

[4] a) X. Feng, V. Marcon, W. Pisula, M. R. Hansen, J. Kirkpatrick, F. Grozema, D. Andrienko, K. Kremer, K. Müllen, Nature Mater. 2009, 8, 421-426; b) D. Wu, L. Zhi, G. J. Bodwell, G. Cui, N. Tsao, K. Müllen, Angew. Chem. Int. Ed. 2007, 46, 5417-5420; c) D. Wu, W. Pisula, V. Enkelmann, X. Feng, K. Müllen, J. Am. Chem. Soc. 2009, 131, 96209621.

[5] a) D. Wang, L. J. Wan, J. Phys. Chem. C 2007, 111, 16109-16130; b) M. Kunitake, N. Batina, K. Itaya, Langmuir, 1995, 11, 2337-2340; c) M. Kunitake, U. Akiba, N. Batina, K. Itaya, Langmuir 1997, 13, 16071615; d) N. T. M. Hai, K. Wandelt, P. Broekmann, J. Phys. Chem. C 2008, 112, 10176-10186; e) K. Cui, O. Ivasenko, K. S. Mali, D. Wu,
X. Feng, K. Müllen, S. De Feyter, S. F. L. Mertens, Chem. Commun. 2014, DOI: $10.1039 / \mathrm{c} 4 \mathrm{cc} 04189 \mathrm{e}$.

[6] C. F. J. Faul, M. Antonietti, Adv. Mater. 2003, 15, 673-683.

[7] a) Y. He, T. Ye, E. Borguet, J. Am. Chem. Soc. 2002, 124, 1196411970; b) P. V. C. Medeiros, G. K. Gueorguiev, S. Stafström, Phys. Rev $B$ 2012, 85, 205423; c) W. D. Wheeler, B. A. Parkinson, Y. Dahnovsky, J. Chem. Phys. 2011, 135, 024702; d) C. B. France, P. G. Schroeder, J. C. Forsythe, B. A. Parkinson, Langmuir 2003, 19, 1274-1281

[8] T. Dretschkow, T. Wandlowski, in Solid-liquid interfaces (Eds: K. Wandelt, S. Thurgate), Springer-Verlag, Berlin, 2003, pp. 259-321.

[9] K. S. Mali, D. Wu, X. Feng, K. Müllen, M. Van der Auweraer, S. De Feyter, J. Am. Chem. Soc. 2011, 133, 5686-5688.

[10] X. Yang, B. Wu, C. Janiak, CrystEngComm 2004, 6, 126-129.

[11] a) X. Gao, A. Hamelin, M. J. Weaver, J. Chem. Phys. 1991, 95, 69936996; b) R. Yamada, K. Uosaki, J. Phys. Chem. B 2000, 104, $6021-$ 6027; c) D. M. Kolb, J. Schneider, Electrochim. Acta 1986, 31, 929936; d) U. W. Hamm, D. Kramer, R. S. Zhai, D. M. Kolb, J. Electroanal. Chem. 1996, 414, 85-89.

[12] R. F. W. Bader, Atoms in Molecules: A Quantum Theory, Oxford University Press, New York, 1990.

[13] M. Wilms, M. Kruft, G. Bermes, K. Wandelt, Rev. Sci. Instrum. 1999, 70, 3641-3650.

[14] J. J. Mortensen, L. B. Hansen, K. W. Jacobsen, Phys. Rev. B 2005, 71, 035109.

[15] J. Enkovaara, et al., J. Phys.: Condens. Matter 2010, 22, 253202.

[16] A. Tkatchenko, M. Scheffler, Phys. Rev. Lett. 2009, 102, 073005.

[17] A. Held, M. Moseler, M. Walter, in preparation. 


\section{Angewandte \\ Communications}

\section{Entry for the Table of Contents}

\section{D Self-Assembly}

Kang Cui, Kunal S. Mali, Oleksandr Ivasenko, Dongqing Wu, Xinliang Feng, Michael Walter, Klaus Müllen, Steven De Feyter, ${ }^{*}$ and Stijn F. L. Mertens*

$$
\text { Page - Page }
$$

Squeezing, then Stacking: from Breathing Pores to 3-Dimensional Ionic Self-Assembly under Electrochemical Control

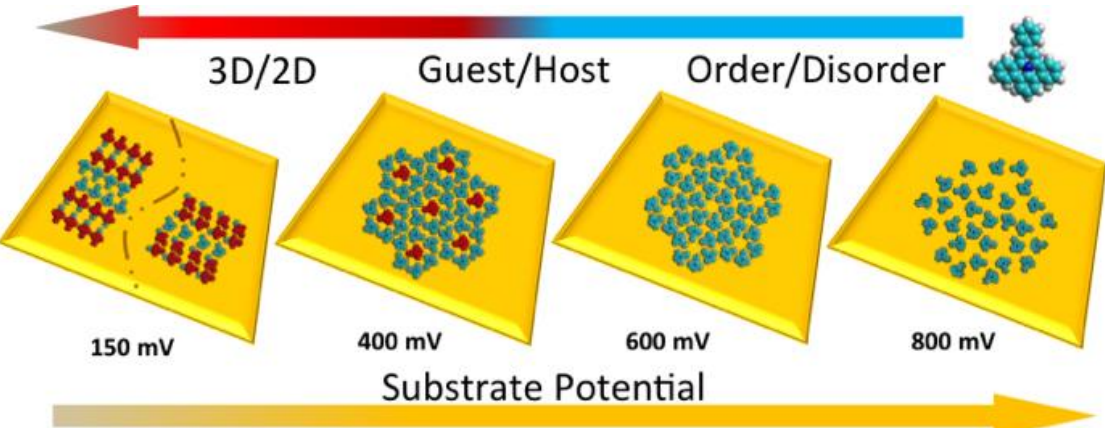

By tuning the substrate potential, a charged discoid polycyclic aromatic compound can be made to self-assemble into open pores, an auto-host-guest structure, or stack into a stratified bilayer. 